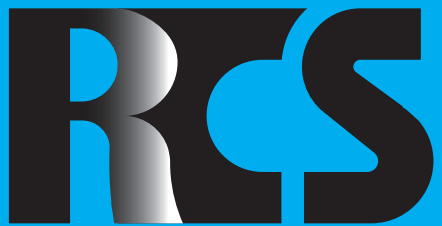

Depósito legal ppi $201502 Z U 4662$

Esta publicación científica en formato

digital es continuidad de la revista impresa

Depósito Legal: pp $197402 Z U 789$

ISSN: 1315-9518

\title{
Revista de Ciencias Sociales
}

Universidad del Zulia. Revista de la Facultad de Ciencias Económicas y Sociales Vol. XXV. Número especial 1, 2019

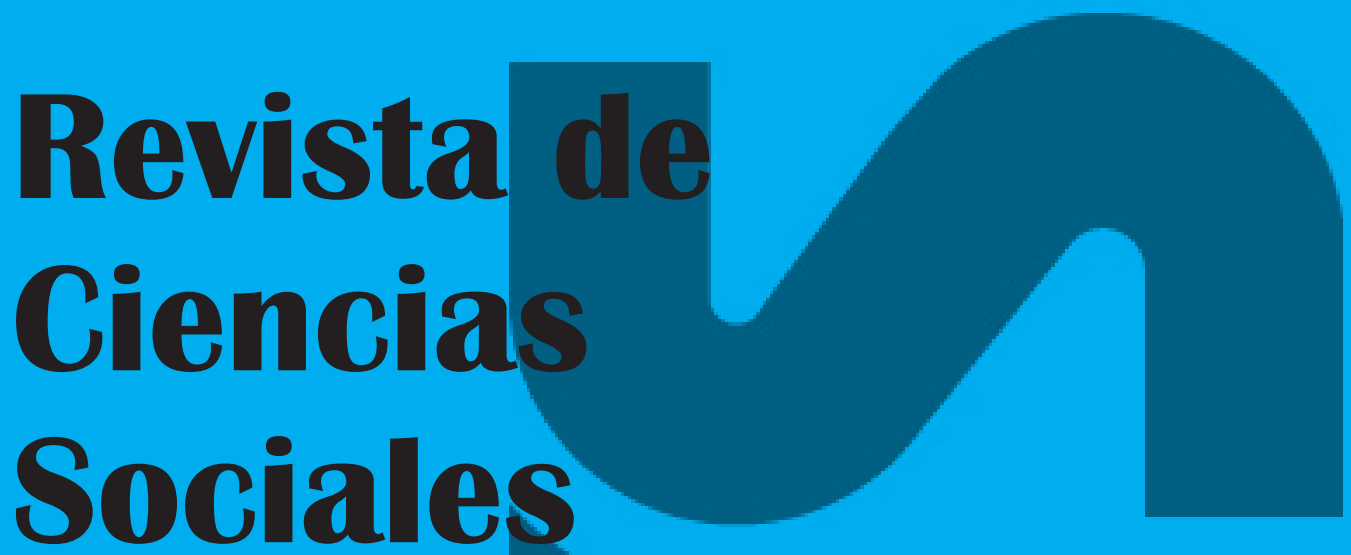




\title{
Percepciones de los hijos únicos sobre sus vivencias en la interacción universitaria
}

\author{
Llanos Rosas, Marcos Gustavo*
}

\section{Resumen}

Los hijos únicos se vinculan mucho a la soledad puesto que no tienen con quien compartir y por lo cual han tenido que desarrollar destrezas de relaciones interpersonales; en el ambiente académico, son uno más entre muchos y por ello pueden tener apremio para su desempeño universitario. El objetivo del presente artículo consiste en estudiar las percepciones de los hijos únicos sobre sus vivencias en su interacción universitaria. Se desarrolló un estudio cualitativo de diseño fenomenológico, en la Facultad de Medicina de la Universidad Nacional de San Agustín Arequipa-Perú, utilizando como unidad de análisis el grupo de hijos únicos del primer a tercer año, a través de focus groups a una muestra no probabilística. Entre los resultados destacan aceptables relaciones con los padres; preocupación por fomentar habilidades de relaciones sociales; así como autonomía y privacidad, en manejo de pertenencias. En el desarrollo escolar, presionados por esperanzas puestas en ellos; muy selectivos en relaciones amicales y responsabilidad de un estándar alto en sus notas educativas. No obstante en la interacción universitaria, al no tener hermanos y por ende insuficiente consulta sobre experiencias previas, trae falta de empatía a pares. Se concluye que los hijos únicos representan un grupo vulnerable y sensitivo para atención académica.

Palabras clave: Atención académica; diseño fenomenológico; grupo focal; hijos únicos; interacción universitaria.

Doctor en Medicina. Magister en Salud Pública. Médico Epidemiólogo del Seguro Social del Perú. Docente de la Facultad de Medicina de la Universidad Nacional San Agustín, Arequipa, Perú. E-mail: mllanosr@unsa.edu.pe 


\title{
Perceptions of unique children about their experiences in university interaction
}

\begin{abstract}
The only children are closely linked to loneliness since they have no one to share and therefore have had to develop interpersonal relationship skills; In the academic environment, they are one more among many and therefore can have pressure for their university performance. The objective of this article is to study the perceptions of the only children about their experiences in their university interaction. A qualitative study of phenomenological design was developed, in the Faculty of Medicine of the National University of San Agustín Arequipa-Peru, using as a unit of analysis the group of single children from the first to third year, through focus groups to a sample not probabilistic The results include acceptable relationships with parents; concern to foster social relations skills; as well as autonomy and privacy, in property management. In school development, pressured by hopes placed on them; very selective in friendly relations and responsibility of a high standard in their educational notes. However, in the university interaction, having no siblings and therefore insufficient consultation about previous experiences, brings lack of empathy to peers. It is concluded that the only children represent a vulnerable and sensitive group for academic attention.
\end{abstract}

Keywords: Academic attention; phenomenological design; focus group; only children; university interaction.

\section{Introducción}

La Educación Superior es aquella que contempla la última fase del proceso de aprendizaje académico, es decir, que viene luego de la etapa secundaria. Es impartida en las Universidades, Institutos Superiores o Academia de Formación Técnica y debe reconocer las diferencias de gusto, ideas, aspecto físico, nacionalidad o religión, pero sin evaluar a uno u otro como mejor. Así Cejas, Rueda, Cayo y Villa (2019) sostienen que "la formación profesional es un proceso que permite preparar o readaptar al individuo para que ejerza un empleo, inicial o no, en una rama cualquiera de la actividad económica" (p.98). Por lo tanto, el paso de la enseñanza media a la universitaria puede significar una vida nueva en otro lugar, alejarse de la familia y amigos, asistir a clases, así como distribuir tiempo y recursos materiales en forma eficiente (Pérez, Valenzuela, Díaz, González y Núñez, 2013).

En las últimas décadas, hubo una disminución progresiva en el tamaño promedio de los hogares, cambio en la estructura familiar y aumento en la frecuencia de familias con apenas un hijo. Históricamente, hay relatos de que el hijo único recibe excesiva atención, madura precozmente y por la ausencia de hermanos, se vuelve egoísta, exigente y temperamental en comparación con los niños con hermanos. Aunque no se confirma el estereotipo del hijo único, lo que sugiere que no tienen problemas de personalidad con más frecuencia que los niños con hermanos e incluso puede tener ventajas relacionadas con la inteligencia, el rendimiento académico y el éxito profesional. Igualmente se ha aludido que la ausencia de hermanos y la mayor convivencia con adultos, pueden interferir en el desarrollo intelectual, la personalidad y la adaptación a la convivencia social (Tavares, et al., 2004).

$\mathrm{Al}$ respecto, la marca del individualismo es uno de los elementos que ocurren en paralelo a la disminución del tamaño de las familias. En 
este sentido, en las familias contemporáneas en las que los niños ocupan la centralidad, el énfasis en la individualización se manifiesta en los arreglos de los hábitos y en los hechos de la familia. En los últimos diez años, por ejemplo en Brasil, hubo un aumento de familias con apenas un hijo. Según datos de la Pesquisa Nacional por Amostra de Domicílio (PNAD), del Instituto Brasileiro de Geografía y Estadística (IBGE, 2015); el número de mujeres con apenas un hijo subió ese año del $25,8 \%$ al $30,7 \%$. Así, el número de hijos ha disminuido, sobre todo en las clases media y media-alta, construyéndose un paralelo entre los cambios de los dinamismos familiares, resultantes de la inserción de la mujer en el mercado de trabajo (Ribeiro y Pedreira, 2011).

Todas las transformaciones sociales y económicas han cambiado la estructura familiar, así también la planificación de los hijos, acrecentando las familias monoparentales con hijos únicos. Ser hijo único desde la perspectiva social, ha sido realmente un problema, por la complicidad de su desarrollo y los calificativos negativos. Cada niño, como ser humano es especial $\mathrm{y}$ único en su momento, estos niños tienen características propias, se adaptan a la forma de educar de su padre y/o madre, a su propia forma de organización y establecen un tipo de vínculo más cercano con su progenitor (Vásquez, 2015).

Al respecto, en el orden de nacimiento de acuerdo a la percepción de los progenitores, los primogénitos fueron descritos como más obedientes, más extrovertidos y menos mimados, si se comparan con menores, mientras que los hijos únicos, fueron descritos como niños menos cautivantes, aunque tenían más disposición estudiosa.

Otros estudios como los de Falbo y Polit (1986), Polit y Falbo (1987) y Mancillas (2006), refuerzan el estereotipo de hijos únicos, como niños con habilidades sociales muchas veces por debajo de lo esperado, con baja autoestima y pocos amigos, además de solitarios, egoístas y dependientes. Sin embargo, poco se sabe sobre las diferencias de prácticas de socialización que afectan a niños con y sin hermanos. El estudio de Rosenberg y Hyde (1993), examinó estas prácticas de madres y padres para con sus hijos, con y sin hermanos, exteriorizando escores mayores en control autoritario y supervisión en los hijos únicos, que en los primogénitos (Freitas y Piccinini, 2010).

Igualmente, hay pocos estudios que se han centrado en el tema de si los diferentes entornos familiares influyen en el desarrollo estructural cerebral de los niños y si el comportamiento tiene de manera diferencial su base neural entre el estado de hijo único y el de hijo no único. Así en el estudio de Yang, et al, 2016; investigaron las diferencias en la cognición (verbi gracia: inteligencia y creatividad) y la personalidad al igual que las diferencias anatómicas estructurales del volumen de materia gris (GMV), utilizando morfometría basada en voxel (VBM) entre hijos únicos y no hijos únicos. Los resultados del comportamiento revelaron que los niños únicos exhibieron puntajes de flexibilidad más altos (una dimensión de la creatividad) y puntuaciones más bajas de amabilidad (una dimensión de los rasgos de la personalidad) que los no hijos únicos.

Lo que es más importante, los resultados de GMV (volumen de materia gris) revelaron que hubo diferencias significativas entre hijos únicos y no únicos que ocurrieron principalmente en las regiones cerebrales del giro supramarginal, que se correlacionó positivamente con los puntajes de flexibilidad; la corteza prefrontal medial (mPFC), que se correlacionó positivamente con las puntuaciones de amabilidad; y la circunvolución parahipocámpica. Estos hallazgos pueden sugerir que el entorno familiar (es decir, único-hijo vs. no-únicohijo), puede jugar un papel importante en el desarrollo del comportamiento y la estructura cerebral de los individuos (Yang, et al., 2016).

Por ello, existiendo desde tiempos pasados y en la actualidad controversias en relación a los comportamientos positivos o negativos que pueden presentar los hijos únicos, siendo este tema poco abordado en el ámbito local, la importancia de este estudio 
radica en dar a conocer si existen rasgos conductuales diferentes en los hijos únicos y su interacción universitaria y poder aportar de esta manera reflexiones y/o conclusiones adecuadas para el acompañamiento en su vida estudiantil; con relevancia en una educación horizontal, que significa considerar a todos los actores del proceso educativo como iguales en su dignidad y derechos, aunque haya diferentes roles entre ellos; con diálogo abierto, cooperación y tolerancia, sin que nadie sea considerado más o menos que el otro, con respeto a cada uno como una persona diferente y valiosa.

En este sentido la presente investigación plantea analizar las percepciones de los hijos únicos sobre sus vivencias en la interacción universitaria, para lo cual se concibe: 1) Indagar sobre las características de crianza de los hijos únicos; 2) Explorar patrones de desarrollo escolar de los hijos únicos; y 3) Analizar las percepciones de interacción universitaria de los hijos únicos.

\section{Metodología}

La presente investigación se considera cualitativa de diseño fenomenológico, en la cual se proyecta describir e inferir los fenómenos desde el punto de vista de cada participante y estructurarlos colectivamente en base a sus experiencias individuales subjetivas en la pesquisa de posibles significados de su interacción como hijos únicos (Mertens, 2005; Hernández, Fernández y Baptista, 2010).

En ese sentido el estudio se realizó en la Facultad de Medicina de la Universidad Nacional de San Agustín (UNSA), que es una entidad estatal de educación superior universitaria establecida el 11 de noviembre de 1828; ubicada en la ciudad de Arequipa. Es la segunda ciudad más poblada del Perú, después de Lima, albergando una población de 960.594 habitantes según los datos del Censo Nacional (Instituto Nacional de Estadística e Informática [INEI], 2017).

La unidad de análisis lo constituyó el grupo de hijos únicos del primer a tercer año de la Facultad de Medicina de la UNSA que cumplieran con los criterios de inclusión, con un número apropiado a una investigación de corte cualitativo. Al considerar este modelo investigativo, la muestra según Hernández, Fernández y Baptista (2010), se puede adecuar al escenario no probabilístico (muestra homogénea, voluntaria) y poder considerar de 5 a 10 casos, en este estudio se logró la aceptación por parte de 8 estudiantes, en concordancia a los tamaños de muestra apropiados a los estudios cualitativos.

En este sentido previa coordinación con la instancia respectiva de la Universidad y considerando los fines de estudio y del carácter anónimo de la información brindada a los participantes, se siguió los pasos establecidos en Hernández, Fernández y Baptista (2010), como sigue a continuación:

- Se determina el número provisional del grupo que puede disminuir o aumentar de acuerdo con el desarrollo del estudio.

- Se define el tipo tentativo de personas que habrán de participar en la sesión. Regularmente durante la inmersión en el campo se percatan del tipo de personas adecuadas para el grupo; pero el perfil también puede modificarse si la investigación así lo requiere.

- Se detectan personas del tipo elegido.

- Se invita a estas personas a la sesión.

- Se organiza la sesión a efectuarse en un lugar confortable, silencioso y aislado. Los participantes deben sentirse "a gusto", tranquilos, despreocupados y relajados. Asimismo, se planea cuidadosamente lo que se va a tratar en la sesión.

- Se lleva a cabo cada sesión. El investigador tiene que crear un clima de confianza (rapport) entre los participantes y no ser percibido como "distante" por los integrantes de la sesión, propiciando la 
intervención ordenada y la interacción entre todos. Durante la sesión se podrá solicitar opiniones, hacer preguntas, administrar cuestionarios, discutir casos, intercambiar puntos de vista y valorar diversos aspectos. Es muy importante que cada sesión se grabe en audio o video (porque así se dispone de mayor evidencia no verbal en las interacciones) y después realizar análisis de contenido y observación. Se debe tener muy en claro la información o los datos que habrán de recolectarse, así como evitar desviaciones del objetivo planteado, aunque se tendrá que ser flexible (vg., si el grupo desvía la conversación hacia un tema que no es de interés para el estudio, dejar que fluya la comunicación, aunque sutilmente retomar los temas importantes para la investigación).

- Se elabora el reporte de sesión, el cual incluye principalmente: Datos sobre los participantes (edad, género, nivel educativo $\mathrm{y}$ todo aquello que sea relevante para el estudio); fecha y duración de la sesión (hora de inicio y terminación), información completa del desarrollo de la sesión, actitud y comportamiento de los participantes hacia el moderador y la sesión en sí, resultados de la sesión; y por último, observaciones del conductor, así como una bitácora de la sesión.

En cuanto a las técnicas, se trabajó con el método de los grupos de enfoque o focal (focus groups), que consistió en la reunión del grupo, en las cuales los participantes conversaron en torno a uno o varios temas en un ambiente relajado e informal, bajo la conducción del investigador, teniendo como objetivo generar y analizar la interacción entre ellos. De acuerdo con Hernández, Fernández y Baptista (2010), los grupos de enfoque se utilizan en la investigación cualitativa en todos los campos del conocimiento, y varían en algunos detalles según el área.

Algo muy importante es que en esta técnica de recolección de datos, la unidad de análisis es el grupo (lo que expresa y construye), por lo cual tiene su origen en las dinámicas grupales, y el formato de las sesiones es parecido a grupos de crecimiento en el desarrollo humano. Se reúne a un grupo de personas y se trabaja con éste en relación con los conceptos, las experiencias, emociones, creencias, categorías, sucesos o los temas que interesan en el planteamiento de la investigación. Lo que se busca es analizar la interacción entre los participantes y cómo se construyen significados grupalmente, a diferencia de las entrevistas cualitativas, donde se busca explorar al detalle las narrativas individuales.

Los grupos de enfoque no sólo tienen potencial descriptivo, sino sobre todo tienen un gran potencial comparativo que es necesario aprovechar (Hernández, Fernández y Baptista, 2010; Murillo y Martínez, 2010). En la planificación del focus group se consideran los siguientes puntos: Saludo, presentación, explicación, actividades, conclusiones, preguntas, despedida.

Por otro lado, según Hernández, Fernández y Baptista (2010), se utilizó un proceso de análisis que incorpora las concepciones de la metodología en el campo cualitativo. En la recolección de datos, la acción esencial consiste en que se reciben datos no estructurados, a los cuales se les da estructura. Los datos son muy variados, pero en esencia gravitan en narraciones de los participantes auditivas (grabaciones), expresiones verbales y no verbales (como respuestas orales y gestos en una entrevista o grupo de enfoque), además de las narraciones del investigador (anotaciones o grabaciones en la bitácora de campo, ya sea una libreta o un dispositivo electrónico).

Una fuente de datos importantísima que se agrega al análisis la constituyen las impresiones, percepciones, sentimientos $\mathrm{y}$ experiencias del investigador (en forma 
de anotaciones o registradas por un medio electrónico), el análisis de los datos no es predeterminado, sino que es "prefigurado, coreografiado o esbozado". Es decir, se comienza a efectuar bajo un plan general, pero su desarrollo va sufriendo modificaciones de acuerdo con los resultados. Dicho de otra forma, el análisis es moldeado por los datos (lo que los participantes o casos van revelando y lo que el investigador va descubriendo). Asimismo, para la ejecución del presente trabajo de investigación previamente se solicitó a los participantes su participación voluntaria a través de un consentimiento informado.

\section{Resultados y discusión}

El objetivo del estudio se focaliza a la comprensión fenomenológica de las vivencias de los hijos únicos en su interacción universitaria y gracias a ello, se pudo comprender a partir de sus narraciones de grupo, el propio mundo de sus relaciones humanas tanto familiares, escolares $\mathrm{y}$ posteriormente en la universidad.
Estos escenarios de desarrollo les ha significado el tener que poner en práctica habilidades relacionales y sociales, poco aprendidas por la ausencia de hermanos, y con ello manejar de la mejor manera conflictos con sus pares, para fomentar relaciones satisfactorias de compañerismo e interacción estudiantil.

\subsection{Reflexiones sobre la relación con los padres}

En este sentido como se puede apreciar en el Cuadro 1, los participantes señalan en su conjunto que las relaciones con sus padres han sido bastante buenas, recibiendo casi toda la atención, disfrutando de predilección a sus inquietudes y recibiendo todo lo que podían brindarles; aunque en varios casos para ciertos deseos o cosas a pedir, estaban condicionadas a cumplir con ciertas normas de conducta para poder recibir el regalo solicitado.

\section{Cuadro 1 \\ Relación con los padres}

\begin{tabular}{|c|c|c|}
\hline TEMAS ESPECÍFICOS & DESCRIPCIÓN & UNIDADES DE SIGNIFICADO \\
\hline & & $\begin{array}{l}\text { No me gustaba que ingresen a mi casa y puedo hacer } \\
\text { lo que yo quiera porque mis padres son solo para } \\
\text { mí y ser siempre la atención solo de ellos siempre } \\
\text { manteniendo un triángulo que llena todo (A04) }\end{array}$ \\
\hline Relaciones de Afinidad & $\begin{array}{l}\text { Las relaciones afectivas } \\
\text { son más intensas con los } \\
\text { padres. }\end{array}$ & $\begin{array}{l}\text { Hasta cierta edad considere que ser hijo único era } \\
\text { lo mejor que me hubiera podido pasar porque todo } \\
\text { lo que nos padres consiguen es para nosotros, es } \\
\text { como que te complacen en todo sintiéndome como } \\
\text { lo máximo a comparación de mis primos que si se } \\
\text { compran algo tienen que comprarlo para todos en } \\
\text { comparación de que solo para mí era el regalo (A02) }\end{array}$ \\
\hline
\end{tabular}




\section{Cont... Cuadro 1}

\section{Relaciones Positivas}

Suelen ser más

estimulados por su padres.
Los hijos únicos son adecuadamente apoyados por sus padres.

Relaciones Negativas
Los padres han apostado y ganado a un solo número $\mathrm{y}$ son dados a tener un cuidado lleno de ansiedad de su orgullo y su alegría.
Quería postular a Medicina pero no porque mis padres fueran médicos sino porque yo sentía que era el sentido de vida para lo que Dios me había preparado, yo le dije Señor que quieres que estudie Él me decía Medicina, pero mis padres me decían para que vas a estudiar Medicina te vas a cansar hay muchos cursos y ellos me indicaban que te vas al extranjero a estudiar por ejemplo negocios pero como mantenía mi decisión entonces mi mamá me dijo ya ni modo y a donde quieres estudiar a la UNSA (A08)

Con la ayuda de un psicólogo aprendí a interactuar con las personas y también con cursos de oratoria donde tenía que hablar con las personas y así hice más amigos (A05)

Me acostumbre a la soledad, llegar a mi casa, ver a mis abuelitos en su cuarto, mi papá trabajaba mi mamá estaba enferma y me volví más introvertida y mi mamá se preocupaba por que no estaba bien que no me relacione y me hizo ingresar al teatro para aprender a relacionarme (A07)

Porque no hay nadie más y también hay ayuda completa y ello te fortalece pero te presiona porque todas las esperanzas están en uno (A01)

Cuando tuve mi enamorada por miedo a que su mamá le obligará a terminar y le dijera que no puedes tener enamorada por no pedirle permiso, fue un día antes a decirle a su mamá si podía tener enamorada y voy a ser responsable voy a hacer todo lo que tú me pidas y recién pude tener mi primer enamorada a los 14 - 15 años (A06)

Fuente: Elaboración propia, 2019.

También reconocen en general la preocupación por parte de sus padres en fomentar habilidades de relaciones sociales, llegando en alguno de los participantes a tener la asesoría psicológica, y en más de uno a la participación en talleres de oratoria para una mejor interacción estudiantil. En una de las participantes, dada su condición acomodada pudo tener lo último en tecnología apenas salía al mercado por la relación paternal en su hogar, pero al igual que la mayoría de los participantes estaba en concordancia a una 
adecuada observancia de éxitos en su actuar.

En contraste a esta unión familiar manifestaron tener una relativa autonomía y privacidad en el manejo de sus pertenencias, y que nadie podía tocarlas sin su permiso. Igualmente relataron que en lo posible sus padres trataron de motivarlos a estudios de calidad llegando a matricularlos en colegios particulares para lograr estos anhelos.

Es de resaltar el comentario de una de las participante "...no me gustaba que ingresen a mi casa y puedo hacer lo que yo quiera porque mis padres son solo para mí y ser siempre la atención solo de ellos siempre manteniendo un triángulo que llena todo" (A04).

En este sentido, el "síndrome del hijo único" según Adler (2003) supone que quienes no tienen hermanos, de pequeños reciben tanta atención de sus padres dando una generación de hijos egocéntricos. Ese hijo único es el primero y último que van a tener. Al ser su única oportunidad para ejercer de padres, se sienten en el deber de hacerlo bien. Por lo general, estas personas se esfuerzan al máximo para darle lo mejor a su hijo, quien a su vez hace todo lo posible por agradar y no defraudar a sus padres.

El hijo único se lleva toda la atención social, emocional y material de los padres. No tiene que compartir con nadie, pero también debe tener la capacidad de absorber todo lo que sus padres le ofrecen. Cuando juegan, la mayoría de las veces lo hacen solitos y son todos los personajes a la vez: El bueno, el malo, el héroe y el perro. Todavía lo son, en cierto modo. Al estar solos y no tener con quien jugar, desarrollan su imaginación y creatividad. Por otro lado, al no tener que competir con sus hermanos por la atención de sus padres, son menos celosos y envidiosos.

El no haber tenido hermanos con quienes pelear o defenderse, impulsa a muchos hijos únicos a asumir comportamientos más tranquilos y a veces más maduros que los llevan a evitar conflictos. En ocasiones, al no tener hermanos, no aprenden a compartir, a jugar ni a socializarse con otros niños. Esto puede hacer que disminuya su madurez emocional y su grado de sociabilidad, puesto que no aprenden a negociar, a discutir, a enojarse, o a desarrollar plenamente su empatía.

La descripción de que los hijos únicos conviven predominantemente con adultos en la infancia, sugiere que son más maduros y de comportamientos adultos más precozmente que los demás hijos con hermanos (Tavares, et al., 2004).

Adler (2003), explica a través de su teoría la importancia de la constelación familiar en el desarrollo de la personalidad, aquí considera el orden de nacimiento tomando en cuenta el caso específico de los hijos únicos. La opción por el menor número de hijos, surge del deseo de tener aquellos a los que se puede atender bien. El aumento de hijos únicos priva a muchos niños de la experiencia del amor fraternal. Estos padres corren el peligro de caer en estilos de educación sobreprotectores (Valdivia, 2008).

La literatura señala como principales elementos en la génesis de la autoestima infantil, la influencia familiar, es decir, el estilo de educación y crianza, así como la influencia escolar, o lo que es lo mismo, el medio académico. Respecto a la fratría, las diferencias sólo son significativas en la infancia, siendo los hijos únicos y los primogénitos los que poseen una autoestima más elevada. En la adolescencia, la fratría ya no es una variable que permita discriminar el nivel de autoestima, superioridad/popularidad y ansiedad/aislamiento en función de la posición que se ocupa dentro del seno de la familia (Navarro, Tomás y Oliver, 2006).

En ese sentido, la crianza de los hermanos/as va a resultar de mayor calidad cuando un niño/a es primogénito/a, cuando el tamaño de la familia es pequeño dos miembros, para los primogénitos/as y pequeños/as, así como al ser de tres miembros, para los medianos/as; cuando la diferencia de edad respecto a su hermano/a anterior y/o posterior es amplia (igual o mayor que tres años) y cuando es el único/a en su sexo.

Lo anterior se debe a que los padres pueden ejercer una mayor cantidad y calidad de interacción con sus hijos/as, lo que se podría denominar potencial de aprovechamiento 
de los recursos de crianza. Los niños/as que cumplían simultáneamente todas o casi todas las condiciones favorables para recibir una adecuada atención de los padres, han sido denominados diferenciados, los que cumplían simultáneamente todas o casi todas las condiciones contrarias a las primeras han sido denominados no diferenciados; y los que cumplían unas condiciones sí y otras no, han sido denominados semi-diferenciados.

Los sujetos calificados como diferenciados, poseen un amplio espacio de autoidentificación porque su estatus fraterno está conformado por variables estructurales o descriptores, que facilitan una mayor cantidad y calidad de interacción con sus hermanos/as y con los padres. Los hijos/as únicos/as, que son cada vez más numerosos, han sido estimados como grupo aparte; su aprovechamiento exclusivo de los recursos educativos parentales permitiría calificarles como hiperdiferenciados, pero carecen de relación con otros hermanos/as, lo cual les confiere historial interactivo intrafamiliar peculiar (Arranz, Yenes, Olabarrieta y Martín, 2001).

De igual manera, está concordado que algo tan insignificante como el número de palabras que un niño oye antes de cumplir los tres años, influye de forma considerable en el desarrollo de su carácter al crecer. No sería, pues, de extrañar que ser hijo único tuviera también alguna repercusión en la forma de ser, mostrándose muy seguros de sí mismos porque están acostumbrados a tratar con adultos. Se sienten cómodos hablando con ellos, porque hasta cierto punto se sitúan a su misma altura (Fernald, Marchman y Weisleder, 2013).

\subsection{Reflexiones sobre el desarrollo escolar de los hijos únicos}

Tal como se puede observar en el Cuadro 2, en la reunión grupal los participantes consideraron que, eran vistos como una inversión y por ello se consideraban presionados al ser único/a y todas las esperanzas están puestas en ellos; igualmente aunque tienen la oportunidad para interrelacionarse con sus compañeros de estudios, lo hacen en cierto sentido aunque lo consideran de relativa importancia, puesto que aprendieron a convivir con su soledad. Pero cuando se relacionan amicalmente con alguien está intimidad por parte de los únicos/as, es si es posible para toda la vida, siendo muy selectivos y formales en este tipo de relación de reciprocidad.

Es casi general la percepción de la responsabilidad gestada en los hijos/as únicos/ as de mantener un estándar alto en sus notas educativas, tal vez en años anteriores para la consecución de un juguete, pero en este escenario del colegio es desarrollado en forma natural y espontánea, aunque como se refiere anteriormente al no tener hermanos les genera un halo de ser la única esperanza de sus padres.

En uno de los participantes el deseo de encajar en su entorno y apegarse a lo que los otros quieren, le hizo tomar malas decisiones juveniles y dejar un poco los estudios, para no estar en conflicto con sus pares. Como relata una de las intervinientes:

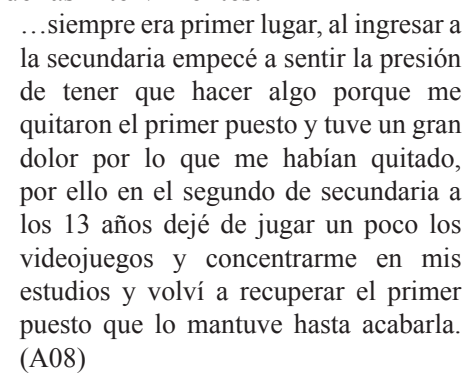

El ambiente brindado por una institución educativa en general es diferente a su hogar, aquí es donde tiene que conllevar con el resto de compañeros y aceptarlos tal como son y no es el cuidado tan sólo para él, sino que es para todos la responsabilidad de educar del colegio. Una escollo que se presenta en los iniciales del plantel es de que los únicos/as saben relacionarse bien con los adultos, pero no están acostumbrados con niños de su propia edad y cuando van al colegio se dan cuenta que son uno entre muchos y que no se trata solo de intercambio de nociones, sino también 


\title{
Cuadro 2
}

\section{Desarrollo escolar de los hijos únicos}

\section{DESCRIPCIÓN}

Adaptación al ambiente escolar, compañeros y nuevos adultos.

\section{Presión por parte de los Padres}

Los padres valoran sobremanera la inteligencia de hijo único.

Hay personas buenas y malas a las que conocer mientras se interactúe con ellas.
Relaciones Positivas

Relaciones Negativas
Las personas piensan que por ser hijo único eres introvertido.

\begin{abstract}
En los estudios ya que tus padres depositan toda su confianza y me consideran como una inversión, cuando tienen hijos son como varios proyectos que nacen y si es que el proyecto resulta es chévere y si no resulta pues si tienes más proyectos tienes la esperanza de que uno de los proyectos resulte, pero cuando tienes un solo proyecto se espera con todas tus ansias de que este proyecto resulte si o si y de alguna manera uno se siente presionado directamente por ello (A01)
\end{abstract}

En relación a los juguetes solo cuando tenía notas de 18 y era premio a que me esforzaba (A03)

Siempre era primer lugar, al ingresar a la secundaria empecé a sentir la presión de tener que hacer algo porque me quitaron el primer puesto y tuve un gran dolor por lo que me habían quitado, por ello en el segundo de secundaria a los 13 años dejé de jugar un poco los videojuegos y concentrarme en mis estudios y volví a recuperar el primer puesto que lo mantuve hasta acabarla (A08)

Desde pequeño jugaba con mis compañeros y sentía que mi profesora era mi segunda mamá llenando el vacío que también tenía en mi casa, estudié en un colegio parroquial donde tenía que estar derechito pasando por un castigo y golpe de los profesores con permiso de los padres y que al final agradecí (A05)

Cuando ingrese a la secundaria recién pude tener una verdadera amiga y la considero como una hermana, los demás para mi eran compañeros (A07)

Particularmente en la primaria considero que en el aspecto social con mis compañeros y docentes fue un poco interrogativa debido a que en la niñez te cuesta más en relacionarte que en la adolescencia y así no me animaba a interactuar por ejemplo con mis compañeros de salón y así era muy introvertido (A02)

Yo al principio fui estudioso hasta el quinto de primaria y ahí empiezan las malas decisiones juveniles y dije quiero ser chévere, estar en salidas, conocer chicas y dejé un poco de lado los estudios y estaba en eso tratando de encajar y por ello tuve que dejar muchas cosas, digamos por tratar de encajar, o te apegas a lo que otros quieren, o si no se da un conflicto y entonces dejas lo que uno desearía hacer (A06)

Fuente: Elaboración propia, 2019.

de emociones. Es un proceso de dar y recibir entre el grupo de compañeros.

En diversos estudios se detectó un mejor desempeño escolar de los hijos únicos en relación a los hijos no primogénitos, más entre los hijos únicos y primogénitos no fue posible caracterizar diferencias independientes y significativas. La asociación inversa entre el orden de nacimiento y desempeño escolar es corroborado por el mejor desempeño escolar de los hijos únicos en diferentes familias y ambientes (Tavares, et al., 2004).

Ungran número de niños son hijosúnicos, creciendo en pequeñas unidades familiares, sin hermanos en casa y tampoco niños del mismo grupo de edad en la vecindad inmediata. Muchos crecen en un ambiente urbano que los priva de libertad de movimientos. Según Bolsanello (2009), la escuela infantil puede ofrecer una atención adecuada mientras los 
padres trabajan, además de un ambiente en el cual los niños puedan pasar sus primeros años aprendiendo y socializándose, gracias a las relaciones que establecen con otros niños y adultos. Esto quizá es debido a la creencia que se tiene de que los hijos únicos poseen menos habilidades sociales. Estudios como el de Miller y Maruyama en 1976, muestran resultados completamente distintos puesto que ellos concluyen que los hijos únicos son menos queridos por sus iguales (Piñeiro, 2016).

\subsection{Reflexiones sobre la interacción universitaria de los hijos únicos}

En el Cuadro 3, los participantes refieren que en su interacción en la universidad ha sido algo complejo, puesto que al no tener hermanos no hay alguien a quién consultarle sobre experiencias previas. Con sus docentes, casi en general no presentan mayor problema por haber crecido en un ambiente de personas mayores, aunque siempre las tratan con bastante respeto. En relación a departir socialmente, no son muy habituados a ello debido a que de pequeños no tuvieron demasiado roce social. En referencia a sentimientos hacia otra persona, especialmente dos participantes manifestaron que está relación les había ayudado a llenar el espacio vacío que sentían en su hogar por la falta de uno de los miembros (en uno de los casos no tenía al padre y en el otro no estaba muy presente porque trabajaba y poco tiempo lo veía), es por ello que se hicieron bastante dependientes de estas relaciones sentimentales.

\section{Cuadro 3 \\ Interacción universitaria de los hijos únicos}

\begin{tabular}{|c|c|c|}
\hline $\begin{array}{c}\text { TEMAS } \\
\text { ESPECÍFICOS }\end{array}$ & DESCRIPCIÓN & UNIDADES DE SIGNIFICADO \\
\hline Con sus Compañeros & $\begin{array}{l}\text { Son más críticos, reflexivos y } \\
\text { observadores pues tuvieron una } \\
\text { interrelación más directa con los } \\
\text { padres y recibiendo una atención } \\
\text { individual y personalizada. }\end{array}$ & $\begin{array}{l}\text { En la Universidad ha sido algo complicado pero no quiero excusarme } \\
\text { de que estoy así porque estoy solo, escuchó que varios compañeros } \\
\text { tienen sus hermanos en años superiores y les dicen que tienen que } \\
\text { hacer en cambio a mi me toca todo nuevo soy mi propio sujeto de } \\
\text { pruebas y al mismo tiempo soy quien reformula, pero tengo que ser } \\
\text { super rápido porque no hay otro que repare en mis acciones (A01) } \\
\text { Normal para conversar con los compañeros, hay fiestas pero no voy, } \\
\text { ya que de pequeño al no ir a ellas no sabría como interactuar (A05) } \\
\text { Cuando acepte ser delegado de un curso, porque varios personajes } \\
\text { querían ser delegados de este curso y como yo siempre deseaba } \\
\text { encajar y ello crea conflictos de interés y ahora si los tenía y parece } \\
\text { que les he quitado algo y así cuando me va mal los ves festejar } \\
\text { cuando me va bien los ves con su mueca (A06) }\end{array}$ \\
\hline Con sus Docentes & $\begin{array}{l}\text { La Universidad, es el sistema en } \\
\text { que puede manifestar su desarrollo } \\
\text { cognitivo, afectivo y social, que } \\
\text { lo ha venido aprendiendo en la } \\
\text { familia. }\end{array}$ & $\begin{array}{l}\text { En la Universidad socializo con mis compañeros pero me cuesta } \\
\text { mucho interrelacionar con los Docentes y personas mayores porque } \\
\text { siento mucho respeto (A02) } \\
\text { La relación con los docentes por razón de haberme criado con } \\
\text { personas de edad no tengo dificultad (A06) } \\
\text { Valoro bastante la amistad en relación a los Docentes no soy de dar } \\
\text { tanta confianza (A07) }\end{array}$ \\
\hline
\end{tabular}




\section{Cont... Cuadro 3}

Relaciones Afectuosas

\begin{abstract}
Lo que es una realidad es que a estar rodeados solo de adultos suelen entrar en la madurez prematuramente y esto les permite desarrollar la destreza que quizá no tengan sus amigos para saber discutir con sus padres y tener excelentes capacidades verbales, dos habilidades que le serán de gran ayuda a la hora de aprender a negociar y conciliar con los demás.
\end{abstract}

Más que nada es el problema emocional que es lo más fundamental puesto que las mujeres son más analíticas y los varones más impulsivos y eso hace que nosotros no lancemos más, también con las cosas del amor, eso hace que te vuelvas un poco sensible, ya que estas acostumbrado a la predilección que te dieron tus padres y crees que el mundo también lo hará pero no es así (A01)

Amor los varones son más lanzados es raro que la mujer tome la iniciativa A partir de os 23 se conocerá el significado del amor, más antes son cambiantes y son las primeras ilusiones (A05)

Me conseguí mi enamorada la actual y no es exactamente una relación de telenovela llega el actor y te quiero para toda la vida y creo que en verdad tengo una dependencia hacia ella por llenar un vació en relación a mi tío (A06)

No conocía a muchas personas, es por ello que en este contexto de fallecimiento de mi abuelo paterno, conocí a una persona en mi clase con la que desarrolle una gran dependencia emocional, era como la luz, me hacía reír y me hacía olvidar lo que me había pasado, pero me hizo daño porque todo hijo único tiene un detalle idealista porque no ha habido mucho roce social y uno espera que las otras personas procedan con la misma seriedad con la que una la está tomando y yo tenía una perspectiva seria en cuanto a las relaciones amicales el compromiso yo estoy ahora contigo y claro tu también estas para mí y yo confié que ella tenía el mismo enfoque de relación amical de compromiso, pero luego me di cuenta que las intenciones de esta persona no eran demasiado buenas y a pesar de tener sólida formación ética moral está persona me estaba haciendo olvidar de ellos que hasta mis padres se dieron cuenta; por lo que Gracias a Dios salí de ella y pude enfocar mejor mis estudios (A08)

\section{Fuente: Elaboración propia, 2019.}

Uno de los participantes refirió: ...en la Universidad ha sido algo complicado pero no quiero excusarme de que estoy así porque estoy solo, escuchó que varios compañeros tienen sus hermanos en años superiores y les dicen que tienen que hacer en cambio a mi me toca todo nuevo soy mi propio sujeto de pruebas y al mismo tiempo soy quien reformula, pero tengo que ser super rápido porque no hay otro que repare en mis acciones. (A01)

A la par otro estudiante refirió: ...cuando acepte ser delegado de un curso, porque varios personajes querían ser delegados de este curso y como yo siempre deseaba encajar y ello crea conflictos de interés y ahora si los tenía y parece que les he quitado algo y así cuando me va mal los ves festejar cuando me va bien los ves con su mueca. (A06)

Otra referencia: ...todo hijo único tiene un detalle idealista porque no ha tenido mucho roce social y uno espera que las otras personas procedan con la misma seriedad con la que una la está tomando y yo tenía una perspectiva seria en cuanto a las relaciones amicales, el compromiso yo estoy ahora contigo y claro tu también estas para mí y yo confié que ella tenía el mismo enfoque de relación amical de compromiso, pero luego me di cuenta que las intenciones de esta persona no eran demasiado buenas y a pesar de tener sólida formación ética moral, está persona me estaba haciendo olvidar de ellos que hasta mis padres se dieron cuenta; por lo que Gracias a Dios salí de ella y pude enfocar mejor mis estudios. (A08)

En cuanto a los factores nointelectuales, una gran proporción de variabilidad escolar están influenciados por elementos que no se pueden medir por medio 
de pruebas de funcionamiento intelectual. La motivación, aparece como uno de los factores no intelectuales que se cree que afectan el rendimiento en el aprendizaje. Las investigaciones y observaciones clínicas de estudiantes con dificultades en los estudios sugieren que hay una variedad de condiciones no Intelectuales que están relacionadas de algún modo con el rendimiento escolar.

Además de la motivación, hay otros factores como Ia adaptación social, familiar y escolar, problemas económicos, clase de escuela superior de donde vienen, problemas de personalidad; que interfieren el éxito universitarito de los estudiantes. Diversas investigaciones han demostrado que estudiantes universitarios que habían tenido experiencias tales como ser hijo único, huérfano, hijo de padres divorciados, graduado de una escuela privada, graduado en una academia militar privada, dos más de las experiencias ya enumeradas, cuando se comparaban con estudiantes universitarios "normales" (quienes no habían tenido ninguna de las experiencias ya dichas) sobre la base de aptitud escolar, probaron que la ejecución del grupo experimental durante su primer semestre universitario fue significativamente inferior en los casos de hijos únicos (Gómez, 1960).

\section{Conclusiones}

Las relaciones con sus padres han sido bastante buenas recibiendo casi toda la atención, siendo un lazo afectivo muy fuerte y arraigado; igual la preocupación por parte de sus padres en fomentar habilidades de relaciones sociales; aunque presentan relativa autonomía y privacidad en el manejo de sus pertenencias.

En el desarrollo escolar se consideraban presionados puesto que todas las esperanzas están puestas en ellos; igualmente son muy selectivos y formales en relaciones de reciprocidad amical y presentan la responsabilidad de mantener un estándar alto en sus notas educativas.

En cuanto a su interacción universitaria, lo consideran algo complejo puesto que al no tener hermanos no hay alguien a quién consultarle sobre experiencias previas, lo que puede traer falta de empatía con sus pares. Aunque con sus docentes no presentaron mayormente problemas.

Igualmente, se puede destacar que desde su infancia pueden tener padres autoritarios o permisivos, lo que posteriormente en sus relaciones amicales $\mathrm{y} / \mathrm{o}$ sentimentales podría ser contraproducente en el sentido a ser demasiado recatados o liberales en estas relaciones; asimismo el no saber cómo podría reaccionar su entorno social hace que su involucramiento gregario se exteriorice a modo de ensayo error; por lo demás en otro contexto pueden pasar momentos de extrema soledad, lo que puede conjugarse hacia escenarios de melancolía, que podría ser tributo para transtornos de ansiedad y/o depresión.

\section{Referencias bibliográficas}

Adler, A. (2003). Psicología individual. En S. Cloninger (Ed.), Teoría de la personalidad (pp. 105-132). México: Pearson Educación.

Arranz, E., Yenes, F., Olabarrieta, F., y Martín, J. L. (2001). Relaciones entre hermanos/as y desarrollo psicológico en escolares. Universidad del País Vasco. Infancia y Aprendizaje, 24(3), 361377.

Bolsanello, M. A. (2009). Prevención desde la escuela infantil: Desafíos en la realidad brasileña. Revista Interuniversitaria de Formación del profesorado, (65), 73-82.

Cejas, M. F., Rueda, M. J., Cayo, L. E., y Villa, L. C. (2019). Formación por competencias: Reto de la educación superior. Revista de Ciencias Sociales (RCS), XXV(1), 94-101.

Falbo, T., y Polit, D. F. (1986). Quantitative review of the only child literature: 
research evidence and theory development. Psychological Bulletin, 100(2), 176-189.

Fernald, A., Marchman, V. A., y Weisleder, A. (2013). SES differences in language processing skill and vocabulary are evident at 18 months. Development Science, 16(2), 234-248.

Freitas, A. P., y Piccinini, C. A. (2010). Práticas educativas parentais em relação ao filho único e ao primogênito. Estudos de Psicología (Campinas), 27(4), 515-528. doi: 10.1590/S0103166X2010000400009

Gómez, F. (1960). Consejo psicológico en el campo universitario. Revista Colombiana de Psicología, 5(1), 51-60.

Hernández, R., Fernández, C., y Baptista, P. (2010). Metodología de la investigación. México D. F.: McGraw-Hill Interamericana Editores..

Instituto Brasileiro de Geografía y Estadística - IBGE (2015). Um registro histórico da Pesquisa Nacional por Amostra de Domicílio (PNAD). Recuperado de https://biblioteca.ibge.gov.br/visualizacao/livros/liv94878.pdf

Instituto Nacional de Estadística e Informática - INEI (2017). Directorio Nacional de Centros Poblados - Censos Nacionales. Recuperado de https://www.inei. gob.pe/media/MenuRecursivo/publicaciones digitales/Est/Lib1541/index. $\underline{\mathrm{htm}}$

Mancillas, A. (2006). Challenging the stereotypes about only children: a review of the literature and implications for practice. Journal of Counseling \& Development, 84(3), 268-275.

Mertens, D. (2005). Research and evaluation in education and psychology: Integrating diversity with quantitative, qualitative, and mixed methods. Thousand Oaks, California: Sage Publications.
Murillo, J., y Martínez, C. (2010). Investigación etnográfica. Métodos de investigación educativa en educación especial. España: Universidad Autónoma de Madrid.

Navarro, E., Tomás, J. M., y Oliver, A. (2006). Factores personales, familiares y académicos en niños y adolescentes con baja autoestima. Boletín de Psicología, (88), 7-25. Recuperado de https:// www.uv.es/seoane/boletin/previos/ N88-1.pdf

Pérez, M. V., Valenzuela, M., Díaz, A., González, J. A., y Núñez, J. C. (2013). Dificultades de aprendizaje en estudiantes universitarios de primer año. Atenea (Concepción), (508), 135-150.

Piñeiro, S. (2016). Diferencias entre hijos únicos e hijos con hermanos en relación con la toma de decisiones, la autoestima, la empatía y el sexo (Tesis de pregrado). Universidad Pontificia de Comillas, España.

Polit, D. F., y Falbo, T. (1987). Only children and personality development: a quantitative review. Journal of Marriage and the Family, 49(2), 309-325.

Ribeiro, L., y Pedreira, E. (2011). Situações familiares na obesidade exógena infantil do filho único. Saúde Soc., 20(2), 507521.

Rosenberg, B. G., y Hyde, J. S. (1993). Differential socialization of only and first-children. Conference Paper presented at Society for Research in Child Development meetings, New Orleans, Louisiana.

Tavares, M. B., Costa, F., Diligenti, F., Pinto, J. R., Rohde, L. A., y Costa, S. (2004). Características de comportamento do filho único vs filho primogênito e não primogênito. Brazilian Journal of Psychiatry, 26(1), 16-22.

Valdivia, C. (2008). La Familia: Concepto, cambios y nuevos modelos. La Revue 
Percepciones de los hijos únicos sobre sus vivencias en la interacción universitaria Llanos Rosas, Marcos Gustavo

du REDIF, 1, 15-22.

Vásquez, V. (2015). Estilos de crianza en familias monoparenterales con hijos únicos (Tesis de maestría). Universidad de Cuenca, Ecuador.

Yang, J., Hou, X., Dongtao, W., Kangcheng,
W., Yadan, L., y Jiang, Q. (2017). Only-child and non-only-child exhibit differences in creativity and agreeableness: Evidence from behavioral and anatomical structural studies. Brain Imaging and Behavior, 11(2), 496-502. doi: 10.1007/s11682-016-9530-9 